\title{
Lyapunov-based backward-horizon adaptive stabilization
}

\author{
Ravinder Venugopal ${ }^{1, *,}$, Venkatesh G. Rao ${ }^{2}$ and Dennis S. Bernstein ${ }^{2}$ \\ ${ }^{1}$ Sysendes Inc., 1804 Rue Tupper, Suite 4, Montreal, QC H3H 1N4, Canada \\ ${ }^{2}$ Department of Aerospace Engineering, The University of Michigan, Ann Arbor, MI 48109-2140, U.S.A
}

\begin{abstract}
SUMMARY
In this paper we develop a discrete-time adaptive stabilization algorithm based on a one-step backwardhorizon cost criterion. By optimizing the cost with respect to the update step size, we obtain a gain update law that guarantees convergence of the plant states. The convergence proof is based on a modified Lyapunov technique. We extend the algorithm to include integral control for rejecting constant disturbances and we present an experimental application to DC motor positioning system. Copyright (C) 2003 John Wiley \& Sons, Ltd.
\end{abstract}

KEY WORDS: Discrete time; Adaptive control; Lyapunov methods

\section{INTRODUCTION}

Although there is no precise definition of adaptive control, one can say intuitively that an adaptive controller operates by adjusting parameters in response to the behaviour of the plant. For continuous-time systems, direct adaptive control algorithms have been developed based on Lyapunov methods [1-5]. The proofs of stability and convergence often depend on the existence of a reference stabilizing controller (called a 'dummy gain matrix' in Reference [3, p. 67]), although knowledge of a stabilizing controller is not needed. For output feedback, these results are generally limited to minimum phase systems with known relative degree.

Direct adaptive control algorithms have also been developed for discrete-time systems [1, 4-13]. However, unlike the continuous-time case, these discrete-time results are based on RLS or LMS algorithms rather than Lyapunov methods. In particular, the approach developed in Reference [6] is based on a convergence result called the Key Technical Lemma (Lemma 6.2.1, pp. 181-182, [12]) which can be applied to RLS or projection-based adaptive control methods. This approach is extended to certain classes of non-minimum phase plants in References [14, 15] and to plants with disturbances in Reference [16]. Extensions of this approach to smooth stabilization with unknown high frequency gain are given in References [17, 18].

\footnotetext{
*Correspondence to: Sysendes Inc., 1804 Rue Tupper, Suite 4, Montreal, QC H3H 1N4, Canada.

†E-mail: rvenugopal@sysendes.com

Contract/grant sponsors: Air Force Office of Scientific Research; contract/grant number: F49620-01-1-0094
} 
Lyapunov synthesis for adaptive control is more straightforward in continuous time because the Lyapunov candidate can usually be chosen such that the derivative is linear in the error states [19]. Nevertheless, Lyapunov techniques have been used for discrete-time direct adaptive control algorithms in References [20-23]. The work in References [20, 21] is based on an RLS approach for model-reference adaptive control in which a cost function based on past inputoutput data is minimized with respect to the current controller parameters. Such an approach is retrospective in the sense that it optimizes controller performance based on past data. A conceptually similar approach is used in Reference [22], where the controller update gradient is based on a window of past data. In Reference [23], a one-step-ahead cost function is used to determine the optimal control signal; however, implementation of this algorithm depends on the choice of two positive-definite matrices that need to satisfy an a priori unverifiable stability condition. In addition, the Lyapunov function for stability analysis of the update law in Reference [23] is based on a parameter identification problem and thus does not explicitly involve the states of the plant and controller.

In this paper, we develop an alternative approach for full-state feedback based on a modified Lyapunov technique and an adaptive step size. We begin by considering an update law for the feedback gain matrix based on minimizing a time-dependent cost function that involves the state at the current time step. The gradient of the cost function with respect to the gain matrix at the previous time step provides an update direction, while the step-size in the gradient direction is chosen such that the distance from the updated gain matrix to the optimal gain is minimized. This optimization is a one-step backward-horizon procedure because the current gain matrix, which affects the state at the next time step, is updated based on the prior cost function involving the current state. An analogous step size is used in References [24-26] within the context of Reference [6], and also in Reference [22] as a key element in an adaptive disturbance rejection algorithm.

We present the main results in Section 2. Implementation issues are discussed in Section 3. In Section 4, we augment the adaptive stablization controller with an integrator to reject step disturbances. Numerical examples with single input and multiple input plants are presented in Section 5, experimental results are given in Section 6, and conclusions are in Section 7.

\section{ADAPTIVE STABILIZATION ALGORITHM}

Consider the discrete-time system

$$
x_{k+1}=A x_{k}+B u_{k}
$$

where $x_{k} \in \mathfrak{R}^{n_{x}}, u_{k} \in \mathfrak{R}^{n_{u}}$ and $k=0,1, \ldots$ denotes the time step. We assume that the pair $(A, B)$ is stabilizable and $\operatorname{rank}(B)=n_{u}$. Furthermore, we assume there exists $K_{\mathrm{S}} \in \mathfrak{R}^{n_{u} \times n_{x}}$ such that $A_{\mathrm{S}} \triangleq A+B K_{\mathrm{s}}$ is asymptotically stable and known. However, we do not assume that we have sufficient knowledge of $A$ and $B$ to actually determine $K_{\mathrm{s}}$. Therefore, our objective is to determine a full-state-feedback control law of the form:

$$
u_{k}=K_{k} x_{k}
$$

such that the origin of the closed-loop system (1), (2) is attractive with respect to $x_{k}$. The adaptive gain matrix $K_{k}$ is updated at each time step $k$ to yield the next gain matrix $K_{k+1}$. 
In certain cases these assumptions can be satisfied with minimal knowledge of the system parameters. For instance, for a single input system in companion form, we do not require knowledge of the last row of $A$. Additional details as well as a multiple input example are given in Section 5.

To derive an adaptively stabilizing control law, we consider the one-step cost function

$$
J_{k}(K) \triangleq \frac{1}{2} \varepsilon_{k}^{T}(K) P \varepsilon_{k}(K)
$$

where $\varepsilon_{k}(K) \triangleq x_{k+1}(K)-x_{k+1}\left(K_{\mathrm{s}}\right), x_{k+1}(K)=(A+B K) x_{k}$, the state at time $k+1$ when the gain matrix $K$ is used at time $k$, and $P \in \mathfrak{R}^{n x n}$ is a positive-definite matrix. Note that $x_{k+1}\left(K_{\mathrm{s}}\right)=A_{\mathrm{s}} x_{k}$. We also define

$$
\hat{J}_{k}(K) \triangleq\left\|K-K_{\mathrm{s}}\right\|_{\mathrm{F}}^{2}
$$

Let $\sigma_{\max }(A)$ denote the maximum singular value of $A$, let $I_{n}$ denote the $n \times n$ identity matrix, and let $Z^{+}$denote the set of nonnegative integers.

\section{Lemma 1}

Consider the gain update law

$$
K_{k+1}\left(\eta_{k}\right)=K_{k}-\left.\eta_{k} \frac{\partial J_{k}}{\partial K}\right|_{K_{k}}=K_{k}-\eta_{k} B^{\mathrm{T}} P \varepsilon_{k}\left(K_{k}\right) x_{k}^{\mathrm{T}}
$$

where $\eta_{k} \in \mathfrak{R}$ and $k \in Z^{+}$. Let $N \in \mathfrak{R}^{n_{x}}$ satisfy $N^{T} N=P$. Then the following statements hold:

(i) If $\varepsilon_{k}\left(K_{k}\right)=0$, then $K_{k+l}\left(\eta_{k}\right)=K_{k}$ for all $n_{k} \in \mathfrak{R}$.

(ii) If $\varepsilon_{k}\left(K_{k}\right) \neq 0$, then $\hat{\eta}_{k}$ given by

$$
\hat{\eta}_{k}=\frac{\left\|N \varepsilon_{k}\left(K_{k}\right)\right\|_{2}^{2}}{\left\|B^{\mathrm{T}} P \varepsilon_{k}\left(K_{k}\right) x_{k}^{\mathrm{T}}\right\|_{\mathrm{F}}^{2}}
$$

is positive and minimizes $\hat{J}_{k}\left(K_{k+1}\left(\eta_{k}\right)\right)$ with minimum value

$$
\left.\hat{\boldsymbol{J}}_{k}\left(K_{k+1}\left(\hat{\eta}_{k}\right)\right)=\hat{\boldsymbol{J}}_{k}\left(K_{k}\right)\right)-\frac{\left\|N \varepsilon_{k}\left(K_{k}\right)\right\|_{2}^{4}}{\left\|B^{T} P \varepsilon_{k}\left(K_{k}\right) x_{k}^{\mathrm{T}}\right\|_{\mathrm{F}}^{2}}
$$

(iii) Suppose $\varepsilon_{k}\left(K_{k}\right) \neq 0$. Then $\hat{J}_{k}\left(K_{k+1}\left(\eta_{k}\right)\right)<\hat{J}_{k}\left(K_{k}\right)$ if and only if $\eta_{k} \in\left(0,2 \hat{\eta}_{k}\right)$. Furthermore, $\hat{J}_{k}\left(K_{k+1}\left(\eta_{k}\right)\right)=\hat{J}_{k}\left(K_{k}\right)$ if and only if either $\eta_{k}=0$ or $\hat{\eta}_{k}=2 \hat{\eta}_{k}$.

Let $\left\{\eta_{k}\right\}_{k \in z^{+}}$be a sequence of positive real numbers, let $K_{0} \in \mathfrak{R}^{n_{u} \times n_{x}}$, let $\left\{K_{k}\right\}_{k=1}^{\infty}$ be the sequence generated by (5), and let $S \triangleq\left\{k \in Z^{+}: \varepsilon_{k}\left(K_{k}\right) \neq 0\right\}$. Then the following statements hold:

(iv) If $S$ is empty, then

$$
\lim _{k \rightarrow \infty} x_{k}=0
$$

(v) If $S$ is not empty and

$$
\sup _{k \in S}\left|\frac{\eta_{k}}{\hat{\eta}_{k}}-1\right|<1
$$


then

$$
\lim _{k \rightarrow \infty} \frac{\left\|N \varepsilon_{k}\left(K_{k}\right)\right\|}{\left\|N x_{k}\right\|}=0
$$

\section{Proof}

To prove (i) let $\varepsilon_{k}\left(K_{k}\right)=0$. Then (5) implies $K_{k+1}\left(\eta_{k}\right)=K_{k}$ for all $\eta_{k}$. To prove (ii), define

$$
\hat{K}_{k} \triangleq K_{k}-K_{\mathrm{s}}
$$

and rewrite (5) as

$$
\hat{K}_{k+1}\left(\eta_{k}\right)=\hat{K}_{k}-\eta_{k} B^{\mathrm{T}} P \varepsilon_{k}\left(K_{s}\right) x_{k}^{\mathrm{T}}
$$

Now using (1), (2) we can write

$$
x_{k+1}\left(K_{k}\right)=\left(A_{\mathrm{s}}+B \hat{K}_{k}\right) x_{k}
$$

which implies

$$
\varepsilon_{k}\left(K_{k}\right)=B \hat{K}_{k} x_{k}
$$

From (12) and (14) it follows that

$$
\begin{aligned}
\hat{J}_{k}\left(K_{k+1}\left(\eta_{k}\right)\right) & =\left\|\hat{K}_{k+1}\left(\eta_{k}\right)\right\|_{\mathrm{F}}^{2} \\
& =\left\|\hat{K}_{k}-\eta_{k} B^{\mathrm{T}} P \varepsilon_{k}\left(K_{k}\right) x_{k}^{\mathrm{T}}\right\|_{\mathrm{F}}^{2} \\
& =\hat{J}_{k}\left(K_{k}\right)+\left\|B^{\mathrm{T}} P B \hat{K}_{k} x_{k} x_{k}^{\mathrm{T}}\right\|_{\mathrm{F}}^{2} \eta_{k}^{2}-2 \operatorname{tr}\left(B^{\mathrm{T}} P B \hat{K}_{k} x_{k} x_{k}^{\mathrm{T}} \hat{K}_{k}^{\mathrm{T}}\right) \eta_{k} \\
& =\hat{J}_{k}\left(K_{k}\right)+\left\|B^{\mathrm{T}} P B \hat{K}_{k} x_{k} x_{k}^{\mathrm{T}}\right\|_{\mathrm{F}}^{2} \eta_{k}^{2}-2\left\|N B \hat{K}_{k} x_{k}\right\|_{2}^{2} \eta_{k} \\
& =\hat{J}_{k}\left(K_{k}\right)+\left\|B^{\mathrm{T}} P \varepsilon_{k}\left(K_{k}\right) x_{k}^{\mathrm{T}}\right\|_{\mathrm{F}}^{2} \eta_{k}^{2}-2\left\|N \varepsilon_{k}\left(K_{k}\right)\right\|_{2}^{2} \eta_{k} \\
& =\hat{J}_{k}\left(K_{k}\right)+\eta_{k}\left(\eta_{k}-2 \hat{\eta}_{k}\right)\left\|B^{\mathrm{T}} P \varepsilon_{k}\left(K_{k}\right) x_{k}^{\mathrm{T}}\right\|_{\mathrm{F}}^{2} \\
& =\hat{J}_{k}\left(K_{k}\right)+\left(\left(\eta_{k}-\hat{\eta}_{k}\right)^{2}-\hat{\eta}_{k}^{2}\right)\left\|B^{\mathrm{T}} P \varepsilon_{k}\left(K_{k}\right) x_{x}^{\mathrm{T}}\right\|_{2}^{2}
\end{aligned}
$$

To minimize $J_{k}\left(K_{k+1}\left(\eta_{k}\right)\right)$, we proceed as follows. By (14), $\varepsilon_{k}\left(K_{k}\right) \neq 0$ implies $\hat{K}_{k} x_{k} \neq 0$ and $x_{k} \neq 0$. Hence $\hat{K}_{k} x_{k} x_{k}^{\mathrm{T}} \neq 0$. Since $B^{\mathrm{T}} P B$ is non-singular, it follows that $\left\|B^{\mathrm{T}} P \varepsilon_{k}\left(K_{k}\right) x_{k}^{\mathrm{T}}\right\|_{\mathrm{F}}^{2}=$ $\left\|B^{\mathrm{T}} P B \hat{K}_{k} x_{k} x_{k}^{\mathrm{T}}\right\|_{\mathrm{F}}^{2} \neq 0$. Therefore $\hat{\eta}_{k}$ can be defined by (6) and $\eta_{k}=\hat{\eta}_{k}$ minimizes $\hat{J}_{k}\left(K_{k+1}\left(\eta_{k}\right)\right)$ with (7).

To prove (iii) assume $\hat{J}_{k}\left(K_{k+1}\left(\eta_{k}\right)\right)-\hat{J}_{k}\left(K_{k}\right)<0$. Then by (15)

$$
\eta_{k}\left(\eta_{k}-2 \hat{\eta}_{k}\right)\left\|B^{\mathrm{T}} P \varepsilon_{k}\left(K_{k}\right) x_{k}^{\mathrm{T}}\right\|_{\mathrm{F}}^{2}<0
$$

which implies $0<\eta_{k}<2 \hat{\eta}_{k}$. Conversely, $0<\eta_{k}<2 \hat{\eta}_{k}$ implies (16), which implies $\hat{J}_{k}\left(K_{k+1}\right.$ $\left.\left(\eta_{k}\right)\right)-\hat{J}_{k}\left(K_{k}\right)<0$ by $(15)$. Setting $\hat{J}_{k}\left(K_{k+1}\left(\eta_{k}\right)\right)=\hat{J}_{k}\left(K_{k}\right)$ in (15) yields $\eta_{k}=0$ or $\eta_{k}=2 \hat{\eta}_{k}$.

To prove (iv) let $\varepsilon_{k}\left(K_{k}\right)=0$ for all $k \in Z^{+}$. This implies $x_{k+1}=A_{s} x_{k}$ for all $k \in Z^{+}$. Since $A_{\mathrm{S}}$ is asymptotically stable, it follows that (8) holds. 
To prove (v), define

$$
\gamma \triangleq \sup _{k \in S}\left|\frac{\eta_{k}}{\hat{\eta}_{k}}-1\right|
$$

By $(9), \gamma<1$, hence $\eta_{k} \in\left[(1-\gamma) \hat{\eta}_{k},(1+\gamma) \hat{\eta}_{k} \subset\left(0,2 \hat{\eta}_{k}\right)\right.$ for all $k \in S$. Hence $\eta_{k} \neq 0$ and $\eta_{k} \neq 2 \hat{\eta}_{k}$. Furthermore, as in the proof of (ii), $\left\|B^{\mathrm{T}} P \varepsilon_{k}\left(K_{k}\right) x_{k}^{\mathrm{T}}\right\|_{\mathrm{F}}^{2} \neq 0$ and $\eta_{k}<2 \hat{\eta}_{k}$. Now let $k \in S$.

Using (6) and (15) we have

$$
\begin{aligned}
\left\|\hat{K}_{k+1}\left(\eta_{k}\right)\right\|_{\mathrm{F}}^{2}-\left\|\hat{K}_{k}\right\|_{\mathrm{F}}^{2} & =\eta_{k}\left(\eta_{k}\left\|B^{\mathrm{T}} P \varepsilon_{k}\left(K_{k}\right) x_{k}^{\mathrm{T}}\right\|_{\mathrm{F}}^{2}-2\left\|N \varepsilon_{k}\left(K_{k}\right)\right\|_{2}^{2}\right) \\
& =\eta_{k}\left(\eta_{k}-2 \hat{\eta}_{k}\right)\left\|B^{\mathrm{T}} P \varepsilon_{k}\left(K_{k}\right) x_{k}^{\mathrm{T}}\right\|_{\mathrm{F}}^{2} \\
& <0
\end{aligned}
$$

Since $S$ is not empty, there exists a positive integer $n>0$ such that $\varepsilon_{k}\left(K_{n}\right) \neq 0$. Let $r_{0}>n$ and, for all $r>r_{0}$, define the non-empty set $S_{r} \triangleq\left\{k: 0 \leqslant k \leqslant r\right.$ and $\left.\varepsilon_{k}\left(K_{k}\right) \neq 0\right\}$. For $r>r_{0}$, it follows from (18) that

$$
\begin{aligned}
\left\|\hat{K}_{0}\right\|_{\mathrm{F}}^{2} & \geqslant\left\|\hat{K}_{0}\right\|_{\mathrm{F}}^{2}-\left\|\hat{K}_{r+1}\right\|_{\mathrm{F}}^{2} \\
& =\sum_{\kappa=0}^{r}\left(\left\|\hat{K}_{k}\right\|_{\mathrm{F}}^{2}-\left\|\hat{K}_{k+1}\right\|_{\mathrm{F}}^{2}\right) \\
& =\sum_{k \in S_{r}} \eta_{k}\left(2 \hat{\eta}_{k}-\eta_{k}\right)\left\|B^{\mathrm{T}} P \varepsilon_{k}\left(K_{k}\right) x_{k}^{\mathrm{T}}\right\|_{\mathrm{F}}^{2} \\
& >0
\end{aligned}
$$

Let $r>r_{0}$, let $k \in S_{r}$, and consider the function $g(\eta)=\eta\left(2 \hat{\eta}_{k}-\eta\right)$ defined on the interval $L=\left[(1-\gamma) \hat{\eta}_{k},(1+\gamma) \hat{\eta}_{k}\right]$. Since $g(\cdot)$ is quadratic, it follows that

$$
\left(1-\gamma^{2}\right) \hat{\eta}_{k}^{2}=\min _{\eta \in L} g(\eta)=g\left((1-\gamma) \hat{\eta}_{k}\right)=g\left((1+\gamma) \hat{\eta}_{k}\right)
$$

Hence,

$$
\eta\left(2 \hat{\eta}_{k}-\eta\right) \geqslant\left(1-\gamma^{2}\right) \hat{\eta}_{k}^{2} \text { for all } \eta \in\left[(1-\gamma) \hat{\eta}_{k},(1+\gamma) \hat{\eta}_{k}\right]
$$

Using (6) and (20), we can rewrite (19) as

$$
\begin{aligned}
\left\|\hat{K}_{0}\right\|_{\mathrm{F}}^{2} & \geqslant\left(1-\gamma^{2}\right) \sum_{k \in S_{r}} \frac{\left.\| N \varepsilon_{k}\left(K_{k}\right)\right) \|_{2}^{4}}{\left\|B^{\mathrm{T}} P \varepsilon_{k}\left(K_{k}\right) x_{k}^{\mathrm{T}}\right\|_{\mathrm{F}}^{2}} \\
& =\left(1-\gamma^{2}\right) \sum_{k \in S_{r}} \frac{\left.\| N \varepsilon_{k}\left(K_{k}\right)\right) \|_{2}^{4}}{\left\|B^{\mathrm{T}} P \varepsilon_{k}\left(K_{k}\right) x_{k}^{\mathrm{T}} N^{\mathrm{T}} N^{-\mathrm{T}}\right\|_{\mathrm{F}}^{2}} \\
& \geqslant\left(1-\gamma^{2}\right) \sum_{k \in S_{r}} \frac{\left.\| N \varepsilon_{k}\left(K_{k}\right)\right) \|_{2}^{4}}{\|N\|_{\mathrm{F}}^{2}\left\|N \varepsilon_{k}\left(K_{k}\right)\right\|_{2}^{2}\left\|N x_{k}\right\|_{2}^{2}\left\|N^{-1}\right\|_{\mathrm{F}}^{2}} \\
& \geqslant\left(1-\gamma^{2}\right) \sum_{k \in S_{r}} \frac{\left.\| N \varepsilon_{k}\left(K_{k}\right)\right) \|_{2}^{2}}{\|N B\|_{\mathrm{F}}^{2}\left\|N x_{k}\right\|_{2}^{2} N^{-1} \|_{\mathrm{F}}^{2}}
\end{aligned}
$$


or

$$
\sum_{k \in S_{r}} \frac{\left\|N \varepsilon_{k}\left(K_{k}\right)\right\|_{2}^{2}}{\left\|N x_{k}\right\|_{2}^{2}} \leqslant \beta
$$

where $\beta \triangleq\left\|\hat{K}_{0}\right\|_{\mathrm{F}}^{2}\|N B\|_{\mathrm{F}}^{2} \mid N^{-1} \|_{\mathrm{F}}^{2} /\left(1-\gamma^{2}\right)$. Letting $r \rightarrow \infty$ yields

$$
\sum_{k \in S} \frac{\left\|N \varepsilon_{k}\left(K_{k}\right)\right\|_{2}^{2}}{\left\|N x_{k}\right\|_{2}^{2}} \leqslant \beta
$$

Next, define the set $S^{\prime} \triangleq Z^{+} \backslash S$ and note that $\varepsilon_{k}\left(K_{k}\right)=0$ for all $k \in S^{\prime}$. If $k \in S^{\prime}$ and $x_{k}=0$ then $x_{l}=0$ for all $l \geqslant k$. Hence assume that $x_{k} \neq 0$ for all $k \in S^{\prime}$. For $k \in S^{\prime}$, we have $\| N \varepsilon_{k} \times$ $\left(K_{k}\right)\left\|_{2}^{2} /\right\| N x_{k} \|_{2}^{2}=0$. Therefore, it follows from (21) that (10) holds.

\section{Theorem 1}

Assume there exists $K_{\mathrm{S}} \in \mathfrak{R}^{n_{u} \times n_{x}}$ such that $A_{\mathrm{S}} \triangleq A+B K_{\mathrm{S}}$ is asymptotically stable, let $R \in \mathfrak{R}^{n_{x} \times n_{x}}$ be positive definite, and let $P \in \Re^{n_{x} \times n_{x}}$ be the positive-definite solution to

$$
P=A_{\mathrm{s}}^{\mathrm{T}} P A_{\mathrm{s}}+R
$$

Let the control be given by (2) with the gain update (5) and with $\left\{\eta_{r}\right\}_{k \in Z^{+}}$satisfying (9). Then

$$
\lim _{k \rightarrow \infty} x_{k}=0
$$

\section{Proof}

If $S$ is empty, the result follows from (iv) of Lemma 1 . Hence assume $S$ is not empty and consider the Lyapunov candidate $V\left(x_{k}\right)=x_{k}^{\mathrm{T}} P x_{k}+\left\|\hat{K}_{k}\right\|_{\mathrm{F}}^{2}$. Then using (22) and (iii) of Lemma 1, we have

$$
\begin{aligned}
V\left(x_{k+1}\right)-V\left(x_{k}\right) & =x_{k+1}^{\mathrm{T}} P x_{k+1}-x_{k}^{\mathrm{T}} P x_{k}+\left\|\hat{K}_{k+1}\right\|_{\mathrm{F}}^{2}-\|\left.\hat{K}_{k}\right|_{\mathrm{F}} ^{2} \\
& \leqslant x_{k+1}^{\mathrm{T}} P x_{k+1}-x_{k}^{\mathrm{T}} P x_{k} \\
& =\left(A_{\mathrm{s}} x_{k}+\varepsilon_{k}\right)^{\mathrm{T}} P\left(A_{\mathrm{s}} x_{k}+\varepsilon_{k}\right)-x_{k}^{\mathrm{T}} P x_{k} \\
& =x_{k}^{\mathrm{T}}\left(A_{\mathrm{s}}^{\mathrm{T}} P A_{\mathrm{s}}-P\right) x_{k}+\varepsilon_{k}^{\mathrm{T}} P \varepsilon_{k}+2 \varepsilon_{k}^{\mathrm{T}} P A_{\mathrm{s}} x_{k} \\
& =-x_{k}^{\mathrm{T}} R x_{k}+\varepsilon_{k}^{\mathrm{T}} P \varepsilon_{k}+2 \varepsilon_{k}^{\mathrm{T}} P A_{\mathrm{s}} x_{k} \\
& \leqslant-x_{k}^{\mathrm{T}} R x_{k}+2\left\|N \varepsilon_{k}\right\|_{2}\left\|N A_{\mathrm{s}} x_{k}\right\|_{2}+\left\|N \varepsilon_{k}\right\|_{2}^{2}
\end{aligned}
$$

where $N \in \mathfrak{R}^{n_{x} \times n_{x}}$ satisfies $N^{\mathrm{T}} N=P$. Thus,

$$
\begin{aligned}
V\left(x_{k+1}\right)-V\left(x_{k}\right) & \leqslant-x_{k}^{\mathrm{T}} R x_{k}+2\left\|N \varepsilon_{k}\right\|_{2}\left\|N A_{\mathrm{S}} N^{-1} N x_{k}\right\|_{2}+\left\|N \varepsilon_{k}\right\|_{2}^{2} \\
& \leqslant-x_{k}^{\mathrm{T}} R x_{k}+2\left\|N \varepsilon_{k}\right\|_{2}\left\|N A_{\mathrm{S}} N^{-1}\right\|_{\mathrm{F}}\left\|N x_{k}\right\|_{2}+\left\|N \varepsilon_{k}\right\|_{2}^{2} \\
& \leqslant-x_{k}^{\mathrm{T}} R x_{k}+2 \sigma_{\max }\left(N A_{\mathrm{S}} N^{-1}\right)\left\|N \varepsilon_{k}\right\|_{2}\left\|N x_{k}\right\|_{2}\left\|N x_{k}\right\|_{2}+\left\|N \varepsilon_{k}\right\|_{2}^{2}
\end{aligned}
$$

Now, since $N^{\mathrm{T}} N=P$, (22) implies

$$
I_{n_{x}}=\hat{A}_{\mathrm{s}}^{\mathrm{T}} \hat{A}_{\mathrm{s}}+\hat{R}
$$


where $\hat{A}_{\mathrm{s}} \triangleq N A_{\mathrm{s}} N^{-1}$ and $\hat{R} \triangleq N^{-\mathrm{T}} R N^{-1}$ is positive definite. Thus, $\sigma_{\max }\left(\hat{A}_{\mathrm{s}}\right)<1$. Therefore,

$$
V\left(x_{k+1}\right)-V\left(x_{k}\right) \leqslant-x_{k}^{\mathrm{T}} R x_{k}+2\left\|N \varepsilon_{k}\right\|_{2}\left\|N x_{k}\right\|_{2}+\left\|N \varepsilon_{k}\right\|_{2}^{2}
$$

Let $\delta>0$. By (v) of Lemma 1 , there exists a positive integer $k_{\delta}$ such that $\left\|N \varepsilon_{k}\right\|_{2} /\left\|N x_{k}\right\|_{2}<\delta$ for all $k>k_{\delta}$. Then for $k>k_{\delta}$ we can write

$$
\begin{aligned}
V\left(x_{k+1}\right)-V\left(x_{k}\right) & <-x_{k}^{\mathrm{T}} R x_{k}+2 \delta\left\|N x_{k}\right\|_{2}^{2}+\delta^{2}\left\|N x_{k}\right\|_{2}^{2} \\
& <-x_{k}^{\mathrm{T}}\left[R-\left(2 \delta+\delta^{2}\right) P\right] x_{k}
\end{aligned}
$$

Now choose $\delta$ sufficiently small such that $R-\left(2 \delta+\delta^{2}\right) P$ is positive definite. Next, for $k>k_{\delta}$, define the translated system

$$
\hat{x}_{\hat{k}+1}=\left(A+B K_{k}\right) \hat{x}_{\hat{k}}, \quad \hat{k} \geqslant 0
$$

where $\hat{k}=k-k_{\delta}$ and $\hat{x}_{\hat{k}} \triangleq x_{k \delta+\hat{k}}$. Using (26), it follows from Theorem 6.3 in Reference [27] that, for the translated system (27) with initial condition $\hat{x}_{0}=x_{k_{\delta}}, \hat{x}_{\hat{k}}^{\mathrm{T}}\left[R-\left(2 \delta+\delta^{2}\right) P\right] \hat{x}_{k} \rightarrow 0$ as $\hat{k} \rightarrow$ $\infty$. Hence $\lim _{\hat{k} \rightarrow \infty} \hat{x}_{k}=0$, and thus $\lim _{k \rightarrow \infty} x_{k}=0$.

The following result provides an alternative step size that guarantees decrease of the cost function $J_{k}$. This result provides a one-step backward horizon interpretation for the gain update law (5).

\section{Proposition 1}

Let $\varepsilon_{k}\left(K_{k}\right) \neq 0$ and define

$$
\eta_{k}^{*}=\frac{\left\|B^{\mathrm{T}} P \varepsilon_{k}\left(K_{k}\right)\right\|_{2}^{2}}{\left\|N B B^{T} P \varepsilon_{k}\left(K_{k}\right) x_{k}^{\mathrm{T}}\right\|_{\mathrm{F}}^{2}}
$$

Then the following statements hold:

(i) $\eta_{k}^{*}$ is positive and minimizes $J_{k}\left(K_{k+1}\left(\eta_{k}\right)\right)$ with minimum value

$$
J_{k}\left(K_{k+1}\left(\eta_{k}^{*}\right)\right)=J_{k}\left(K_{k}\right)-\frac{x_{k}^{\mathrm{T}} x_{k}\left\|B^{\mathrm{T}} P \varepsilon_{k}\left(K_{k}\right)\right\|_{2}^{4}}{2\left\|N B B^{\mathrm{T}} P \varepsilon_{k}\left(K_{k}\right) x_{k}^{\mathrm{T}}\right\|_{\mathrm{F}}^{2}}
$$

(ii) $J_{k}\left(K_{k+1}\left(\eta_{k}\right)\right)<J_{k}\left(K_{k}\right)$ if and only if $\eta_{k} \in\left(0,2 \eta_{k}^{*}\right)$. Furthermore, $J_{k}\left(K_{k+1}\left(\eta_{k}\right)\right)=J_{k}\left(K_{k}\right)$ if and only if either $\eta_{k}=0$ or $\eta_{k}=2 \eta_{k}^{*}$.

(iii) If $\varepsilon_{k}\left(K_{k}\right) \neq 0$, then $\eta_{k}^{*} \leqslant \hat{\eta}_{k}$.

(iv) If $\varepsilon_{k}\left(K_{k}\right) \neq 0$ and $n_{u}=1$, then $\eta_{k}^{*}=\hat{\eta}_{k}$.

\section{Proof}

To prove (i), use (14) to write

$$
J_{k}\left(K_{k}\right)=\frac{1}{2} x_{k}^{\mathrm{T}} \hat{K}_{k}^{\mathrm{T}} B^{\mathrm{T}} P B \hat{K}_{k} x_{k}
$$


Using (12) and (14) we obtain

$$
\begin{aligned}
J_{k}\left(K_{k+1}\left(\eta_{k}\right)\right) & =\frac{1}{2} x_{k}^{\mathrm{T}} \hat{K}_{k+1}\left(\eta_{k}\right)^{\mathrm{T}} B^{\mathrm{T}} P B \hat{K}_{k+1}\left(\eta_{k}\right) x_{k} \\
& =J_{k}\left(K_{k}\right)+\frac{1}{2}\left(x_{k}^{\mathrm{T}} x_{k}\right)^{2}\left[x_{k}^{\mathrm{T}} \hat{K}_{k}^{\mathrm{T}}\left(B^{\mathrm{T}} P B\right)^{3} \hat{K}_{k} x_{k}\right] \eta_{k}^{2}-x_{k}^{\mathrm{T}} x_{k}\left[x_{k}^{\mathrm{T}} K_{k}^{\mathrm{T}}\left(B^{\mathrm{T}} P B\right)^{2} \hat{K}_{k} x_{k}\right] \eta_{k} \\
& \left.=J_{k}\left(K_{k}\right)+\frac{1}{2} x_{k}^{\mathrm{T}} x_{k}\left\|N B B^{\mathrm{T}} P B \hat{K}_{k} x_{k} x_{k}^{\mathrm{T}}\right\|_{\mathrm{F}}^{2} \eta_{k}^{2}-x_{k}^{\mathrm{T}} x_{k} \| B^{\mathrm{T}} P B \hat{K}_{k} x_{k}\right] \|_{2}^{2} \eta_{k} \\
& =J_{k}\left(K_{k}\right)+\frac{1}{2} x_{k}^{\mathrm{T}} x_{k}\left(\eta_{k}-2 \eta_{k}^{*}\right)\left\|N B B^{\mathrm{T}} P \varepsilon_{k}\left(K_{k}\right) x_{k}^{\mathrm{T}}\right\|_{\mathrm{F}}^{2} \\
& =J_{k}\left(K_{k}\right)+\frac{1}{2} x_{k}^{\mathrm{T}} x_{k}\left[\left(\eta_{k}-\eta_{k}^{*}\right)^{2}-\eta_{k}^{* 2}\right]\left\|N B B^{\mathrm{T}} P \varepsilon_{k}\left(K_{k}\right) x_{k}^{\mathrm{T}}\right\|_{\mathrm{F}}^{2}
\end{aligned}
$$

As in the proof of Lemma 1, part (ii), it follows that $\eta_{k}^{*}$ globally minimizes (31) and satisfies (29)

The proof of (ii) is analogous to the proof of Lemma 1, part (iii).

To prove (iii), let $C=\left[\begin{array}{ll}v & B^{\mathrm{T}} P B v\end{array}\right]^{\mathrm{T}}$, where $v \triangleq\left(B^{\mathrm{T}} P B\right)^{1 / 2} \hat{K}_{k} x_{k}$. Since $\operatorname{det}\left(C C^{T}\right) \geqslant 0$ we have

$$
v^{\mathrm{T}} v\left[v^{\mathrm{T}}\left(B^{\mathrm{T}} P B\right)^{2} v\right]-\left(v^{\mathrm{T}} B^{\mathrm{T}} P B v\right)^{2} \geqslant 0
$$

Since $\varepsilon_{k}\left(K_{k}\right) \neq 0$, it follows that $\hat{K}_{k} x_{k} \neq 0, x_{k} \neq 0$ and $v \neq 0$. Therefore,

$$
\eta_{k}^{*}=\frac{v^{\mathrm{T}} B^{\mathrm{T}} P B v}{x_{k}^{\mathrm{T}} x_{k} v^{\mathrm{T}}\left(B^{\mathrm{T}} P B\right)^{2} v} \leqslant \frac{v^{\mathrm{T}} v}{x_{k}^{\mathrm{T}} x_{k} v^{\mathrm{T}} B^{\mathrm{T}} P B v}=\hat{\eta}_{k}
$$

To prove( iv), let $n_{u}=1$. Then $B^{\mathrm{T}} P B$ is a scalar and (33) holds with equality.

\section{Remark 1}

Note that $K_{k+1}$ is computed using the knowledge of $x_{k}$ and $x_{k+1}$ at time $k+1$. The updated gain $K_{k+1}$ is used to propagate the state from $x_{k+1}$ to $x_{k+2}$.

To compute the updated gain matrix $K_{k+1}$ we need the gradient direction of the cost function $J_{k}$ as well as a step size $\eta_{k}$ to move along this direction. To compute the step size $\eta_{k}$ that minimizes the current cost $J_{k+1}\left(K_{k+1}\right)$, it can be seen from the definition of $J_{k}$ and $\varepsilon_{k}$ that we require knowledge of the state $x_{k+2}$ at time $k+1$. Since $x_{k+2}$ is not available at time $k+1$, we instead minimize the prior cost $J_{k}\left(K_{k+1}\right)$ with respect to the updated gain matrix $K_{k+1}$. However, the prior cost $J_{k}\left(K_{k}\right)$ has already been incurred by using $K_{k}$ to move from $x_{k}$ to $x_{k+1}$. Therefore minimizing $J_{k}\left(K_{k+1}\right)$ is a one-step backward horizon cost optimization. Note that $\eta_{k}^{*}$ may not satisfy (9), and thus, there is no guarantee of (23). Theorem 1 guarantees stability for an open interval around the larger step size $\hat{\eta}_{k}$ which minimizes the norm of the distance between $K_{k+1}$ and $K_{\mathrm{s}}$. The relation between the step sizes is shown in Figure 1.

\section{IMPLEMENTATION}

As an application of Theorem 1, consider the single-input system in companion form

$$
A=\left[\begin{array}{cc}
0_{\left(n_{x}-1\right) \times 1} & I_{n_{x}-1} \\
a &
\end{array}\right], \quad B=\left[\begin{array}{c}
0_{\left(n_{x}-1\right) \times 1} \\
b
\end{array}\right]
$$




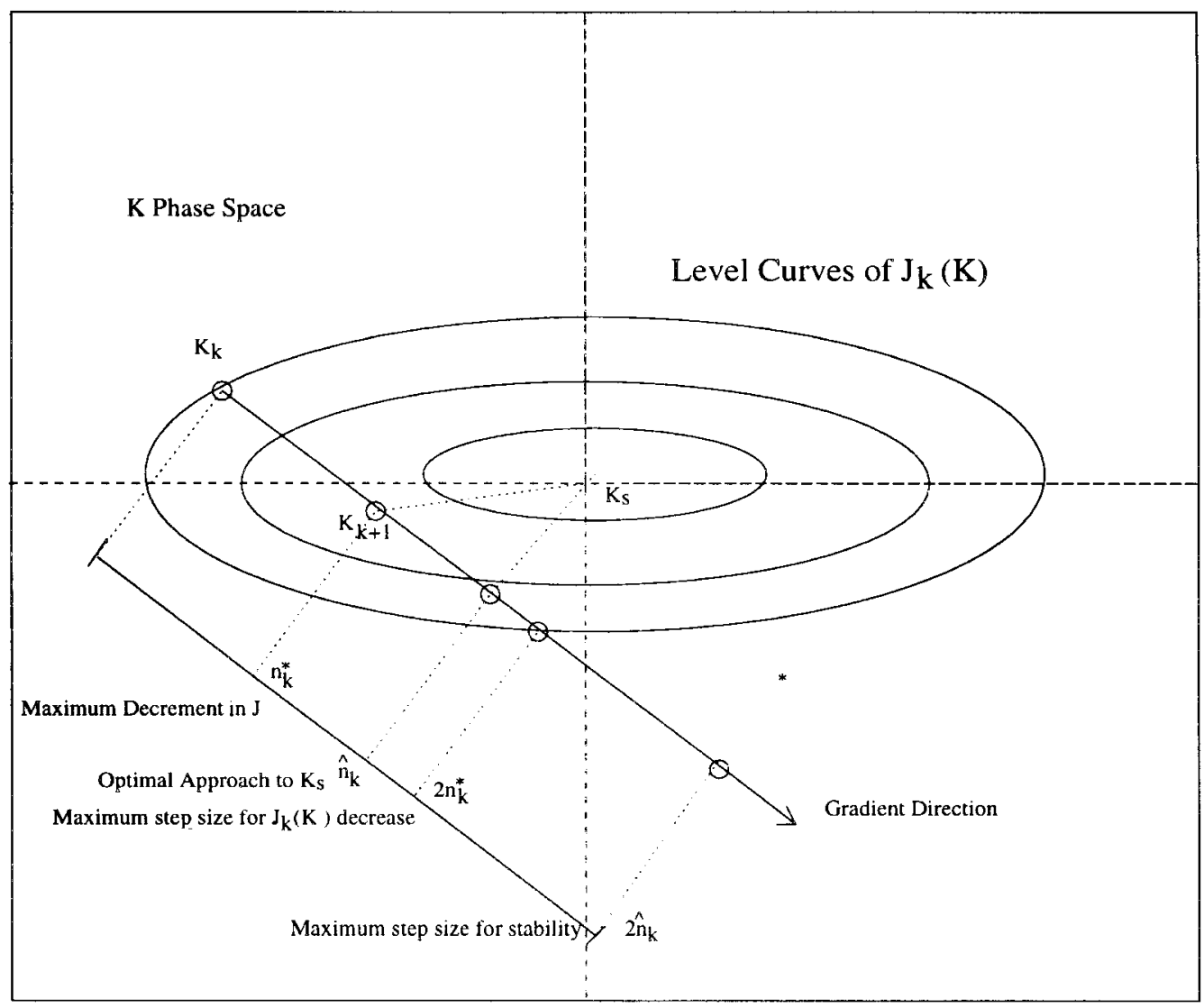

Figure 1. Schematic diagram of the step sizes $\eta_{k}^{*}$ and $\hat{\eta}_{k}$ in terms of the costs $J_{k}$ and $\hat{\boldsymbol{J}}_{k}$.

where $a \in \mathfrak{R}^{1 \times n_{x}}$ and $b \neq 0 \in \mathfrak{R}$. Define

$$
B_{0} \triangleq\left[\begin{array}{c}
0_{\left(n_{x}-1\right) \times 1} \\
\operatorname{sign} b
\end{array}\right]
$$

Letting $K_{\mathrm{S}}=\frac{1}{b}\left(a_{\mathrm{S}}-a\right)$, where $a_{\mathrm{s}} \in \mathfrak{R}^{1 \times n_{x}}$, it follows that

$$
A_{\mathrm{s}}=A+B K_{\mathrm{s}}=\left[\begin{array}{c}
A_{0} \\
a
\end{array}\right]+\left[\begin{array}{l}
0 \\
b
\end{array}\right] \frac{1}{b}\left(a_{\mathrm{s}}-a\right)=\left[\begin{array}{c}
A_{0} \\
a_{\mathrm{s}}
\end{array}\right]
$$

where $a_{\mathrm{s}}$ is chosen such that $A_{\mathrm{s}}$ is asymptotically stable. Since the choice of $a_{\mathrm{s}}$ does not depend on knowledge of either $a$ or $b$, it follows that the solution $P$ of the Lyapunov equation (22) can be determined without knowledge of either $a$ or $b$.

Similarly, we can implement the control law (2), (5) without knowledge of $K_{\mathrm{s}}$ for systems with decoupled inputs. We require knowledge of the rows of $A$ that are not assignable by an input. We also require that $B$ be of the form $B=|b| B_{0}$, where $B_{0}$ is known. An example of such a 
system is the double companion form with decoupled inputs and coupled states

$$
A=\left[\begin{array}{ccc}
0_{n \times 1} & I_{n} & 0_{n \times(n+1)} \\
& a_{1} & \\
0_{n \times 1} & I_{n} & 0_{n \times(n+1)} \\
& a_{2} &
\end{array}\right] B=|b|\left[\begin{array}{cc}
0_{n-1 \times 2} \\
1 & 0 \\
0_{n-1 \times 2} \\
0 & 1
\end{array}\right]
$$

where $a_{1}, a_{2} \in \mathfrak{R}^{1 \mathrm{l}(2 n+2)}$. This system can be stabilized without knowledge of the row vectors $a_{1}, a_{2}$ or the matrix $K_{\mathrm{s}}$.

\section{INTEGRAL CONTROL}

Integral control for rejecting constant disturbances can be incorporated into the algorithm as follows. Consider the closed-loop system

$$
\begin{gathered}
x_{k+1}=A_{x_{k}}+B u_{k}+d \\
u_{k}=K_{k} x_{k}+v_{k}
\end{gathered}
$$

where $d \in \mathfrak{R}^{n}$ is an unknown constant disturbance. Assume that there exists $K_{\mathrm{s}}$ such that $A_{\mathrm{S}} \triangleq A+B K_{\mathrm{S}}$ is asymptotically stable, and also that there exists $v_{\mathrm{s}}$ such that $B v_{\mathrm{s}}=-d$.

From (37) it follows that

$$
u_{k}=\tilde{K}_{k} \tilde{x}_{k}
$$

where $\tilde{K}_{k} \triangleq\left[\begin{array}{ll}K_{k} & v_{k}\end{array}\right]$ and $x_{k} \triangleq\left[\begin{array}{ll}x_{k}^{\mathrm{T}} & 1\end{array}\right]^{\mathrm{T}}$, and thus the closed-loop system can be written as

$$
\tilde{x}_{k+1}\left(\tilde{K}_{k}\right)=\left(\tilde{A}+\tilde{B} \tilde{K}_{k}\right) \tilde{x}_{k}+\tilde{d}
$$

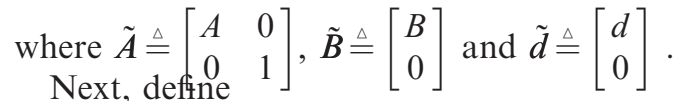

$$
\varepsilon_{k}\left(\tilde{K}_{k}\right) \triangleq \tilde{x}_{k+1}\left(\tilde{K}_{k}\right)-\left(\tilde{A}_{\mathrm{s}} x_{k}+\tilde{d}\right)
$$

where $\tilde{A}_{\mathrm{s}} \triangleq \tilde{A}+\tilde{B} \tilde{K}_{\mathrm{s}}$ with $\tilde{K}_{\mathrm{s}} \triangleq\left[\begin{array}{ll}K_{\mathrm{S}} & v_{\mathrm{s}}\end{array}\right]$. Note that

$$
\varepsilon_{k}\left(\tilde{K}_{k}\right)=\left[\begin{array}{c}
x_{k+1}(K)-A_{\mathrm{s}} x_{k} \\
0
\end{array}\right]
$$

which can be calculated using $A_{\mathrm{s}}$ and a measurement of $x_{k}$.

To derive the adaptive constant disturbance rejection law, we define the cost functions

$$
\begin{gathered}
J_{k}\left(\tilde{K}_{k}\right) \triangleq \frac{1}{2} \varepsilon_{k}^{\mathrm{T}}\left(\tilde{K}_{k}\right) \tilde{P}_{\varepsilon_{k}}\left(\tilde{K}_{k}\right) \\
\tilde{J}_{k}\left(\tilde{K}_{k}\right) \triangleq\left\|\tilde{K}_{k}-\tilde{K}_{\mathrm{s}}\right\|_{\mathrm{F}}^{2}
\end{gathered}
$$

where $P$ is positive definite. 
Lemma 2 Consider the gain update law

$$
\tilde{K}_{k+1}\left(\eta_{k}\right)=\tilde{K}_{k}-\left.\eta_{k} \frac{\partial J_{k}}{\partial \tilde{K}}\right|_{\tilde{K}_{k}}=\tilde{K}_{k}-\eta_{k} \tilde{B}^{\mathrm{T}} \tilde{P}_{\varepsilon_{k}}\left(\tilde{K}_{k}\right) \tilde{x}_{\mathrm{k}}^{\mathrm{T}}
$$

where $\eta_{k} \in \mathfrak{R}$ and $k \in Z^{+}$. Let $\tilde{N}$ satisfy $\tilde{N}^{\mathrm{T}} \tilde{N}=\tilde{P}$. Then the following statements hold:

(i) If $\varepsilon_{k}\left(\tilde{K}_{k}\right)=0$, then $\tilde{K}_{k+1}\left(\eta_{k}\right)=\tilde{K}_{k}$ for all $\eta_{k} \in \mathfrak{R}$.

(ii) If $\varepsilon_{k}\left(\tilde{K}_{k}\right) \neq 0$, then $\hat{\eta}_{k}$ given by

$$
\hat{\eta}_{k}=\frac{\left\|\tilde{N} \varepsilon_{k}\left(\tilde{K}_{k}\right)\right\|_{2}^{2}}{\left\|\tilde{B}^{\mathrm{T}} \tilde{P} \varepsilon_{k}\left(\tilde{K}_{k}\right) \tilde{x}_{k}^{\mathrm{T}}\right\|_{\mathrm{F}}^{2}}
$$

is positive and minimizes $\hat{J}_{k}\left(\tilde{K}_{k+1}\left(\hat{\eta}_{k}\right)\right)$ with

$$
\hat{J}_{k}\left(\tilde{K}_{k+1}\left(\hat{\eta}_{k}\right)\right)=\hat{J}_{k}\left(\tilde{K}_{k}\right)-\frac{\left\|\tilde{N} \varepsilon_{k}\left(\tilde{K}_{k}\right)\right\|_{2}^{4}}{\left\|\tilde{B}^{\mathrm{T}} P \varepsilon_{k}\left(\tilde{K}_{k}\right) \tilde{x}_{k}^{\mathrm{T}}\right\|_{\mathrm{F}}^{2}}
$$

(iii) Suppose $\varepsilon_{k}\left(\tilde{K}_{k}\right) \neq 0$. Then $\hat{J}_{k}\left(\tilde{K}_{k+1}\left(\eta_{k}\right)\right)<\hat{J}_{k}\left(\tilde{K}_{k}\right)$ if and only if $\eta_{k} \in\left(0,2 \hat{\eta}_{k}\right)$. Furthermore, $\hat{J}_{k}\left(\tilde{K}_{k+1}\left(\eta_{k}\right)\right)=\hat{J}_{k}\left(\tilde{K}_{k}\right)$ if and only if either $\eta_{k}=0$ or $\eta_{k}=2 \hat{\eta}_{k}$.

Let $\left\{\eta_{k}\right\}_{k \in z^{+}}$be a sequence of positive real numbers, let $\tilde{K}_{0} \in \mathfrak{R}^{n_{u} \times n_{x}}$. let $\left\{\tilde{K}_{k}\right\}_{k=1}^{\infty}$ be the sequence generated by (5), and let $S \triangleq\left\{k \in Z^{+}: \varepsilon_{k}\left(\tilde{K}_{k}\right) \neq 0\right\}$. Then the following statements hold:

(iv) if $S$ is nor empty and

$$
\sup _{k \in S}\left|\frac{\eta_{k}}{\hat{\eta}_{k}}-1\right|<1
$$

then

$$
\lim _{k \rightarrow \infty} \frac{\left\|\tilde{N} \varepsilon_{k}\left(\tilde{K}_{k}\right)\right\|_{2}}{\|\tilde{N} \tilde{x} k\|_{2}}=0
$$

\section{Proof}

The proof is identical to the proof of Lemma 1.

\section{Theorem 2}

Assume there exists $K_{\mathrm{S}} \in \mathfrak{R}^{n_{u} \times n_{x}}$ such that $A_{\mathrm{S}} \triangleq A+B K_{\mathrm{s}}$ is asymptotically stable, let $\mathfrak{R} \in \mathfrak{R}^{n_{x} \times n_{x}}$ be possible definite, and let $P \in \mathfrak{R}^{n_{x} \times n_{x}}$ be the positive-definite solution to

$$
P=A_{\mathrm{s}}^{\mathrm{T}} P A_{\mathrm{s}}+R
$$

Define $\tilde{P} \triangleq\left[\begin{array}{cc}P & 0 \\ 0 & \lambda^{2}\end{array}\right]$ with $\lambda>0$. Let the control be given by (38) with gain update (44) and with $\left\{\eta_{k}\right\}_{k \in z^{+}}$satisfying (47). Then

$$
\lim _{k \rightarrow \infty} x_{k}=0
$$

Proof

From (48) in Lemma 2, it follows that for all $\delta>0$, there exists a positive integer $l_{\delta}$ such that

$$
\frac{\left\|\tilde{N} \varepsilon_{k}\left(\tilde{K}_{k}\right)\right\|_{2}}{\left\|\tilde{N} \tilde{x}_{k}\right\|_{2}}<\delta
$$


for all $k>l_{\delta}$. Using (41), (51) and the definition of $\tilde{N}$, it follows that

$$
\left\|\left[\begin{array}{cc}
N & 0 \\
0 & \lambda
\end{array}\right]\left[\begin{array}{c}
x_{k+1}-A_{\mathrm{s}} x_{k} \\
0
\end{array}\right]\right\|_{2}<\delta\left\|\left[\begin{array}{cc}
N & 0 \\
0 & \lambda
\end{array}\right]\left[\begin{array}{c}
x_{k} \\
1
\end{array}\right]\right\|_{2}
$$

or

$$
\left\|\left[\begin{array}{c}
N x_{k+1}-N A_{\mathrm{s}} N^{-1} N x_{k} \\
0
\end{array}\right]\right\|_{2}<\delta\left\|\left[\begin{array}{c}
N x_{k} \\
\lambda
\end{array}\right]\right\|_{2}
$$

Thus,

$$
\left\|\left[\begin{array}{c}
N x_{k+1}-\hat{A}_{\mathrm{s}} N x_{k} \\
0
\end{array}\right]\right\|_{2}<\delta \sqrt{\left\|N x_{k}\right\|_{2}^{2}+\lambda^{2}}<\delta\left(\left\|N x_{k}\right\|_{2}+\lambda\right)
$$

where, $\hat{A}_{\mathrm{s}} \triangleq N A_{\mathrm{s}} N^{-1}$, or

$$
\left\|N x_{k+1}-\hat{A}_{\mathrm{s}} N x_{k}\right\|_{2}<\delta\left(\left\|N x_{k}\right\|_{2}+\lambda\right)
$$

Next, define

$$
\mu \triangleq \delta+\sigma_{\max }\left(\hat{A}_{\mathrm{s}}\right)
$$

and note from (49) that $\sigma_{\max }\left(\hat{A}_{\mathrm{s}}\right)<1$. We choose $\delta$ such that $\mu<1$, and thus, from (55), it follows that, for all $r \in Z^{+}$,

$$
\left\|N x_{k+r}\right\|_{2}<\mu^{r}+\delta \lambda \sum_{j=0}^{r-1} \mu^{j}
$$

Thus,

$$
\limsup _{r \rightarrow \infty}\left\|N x_{k+r}\right\|_{2} \leqslant \frac{\delta \lambda}{1-\mu}
$$

for all $\delta>0$ such that $\mu<1$ and for all $\lambda>0$. Hence,

$$
\limsup _{r \rightarrow \infty}\left\|N x_{k+r}\right\|_{2}=0
$$

which implies that

$$
\lim _{r \rightarrow \infty}\left\|N x_{k+r}\right\|_{2}=0
$$

and thus we obtain (50).

\section{NUMERICAL EXAMPLES}

In this section we illustrate the adaptive stabilization algorithm by means of a numerical example. We consider a two-input example. At time $k=30$ the matrix $A$ changes from $A_{1}$, which is open-loop stable, to $A_{2}$, which is open-loop unstable. The controller is unaware of this change. 
The system is given by

$$
\begin{gathered}
A_{1}=\left[\begin{array}{cccc}
0 & 1.0000 & 0 & 0 \\
-0.1634 & -0.1443 & 0.0294 & -0.0140 \\
0 & 0 & 0 & 1.0000 \\
-0.2261 & -0.0585 & -0.1165 & -0.1793
\end{array}\right], \quad B=\left[\begin{array}{ll}
0 & 0 \\
1 & 0 \\
0 & 0 \\
0 & 1
\end{array}\right] \\
A_{2}=\left[\begin{array}{cccc}
0 & 1.0000 & 0 & 0 \\
0.2486 & -1.4897 & 0.3135 & -2.0251 \\
0 & 0 & 0 & 1.0000 \\
0.5290 & 0.3435 & 0.7582 & -0.6919
\end{array}\right] \\
A_{\mathrm{s}}=\left[\begin{array}{cccc}
0 & 1.0000 & 0 & 0 \\
0.2000 & 0.2000 & 0.1300 & 0.1600 \\
0 & 0 & 0 & 1.0000 \\
0.1000 & 0.1500 & 0.3000 & 0.3000
\end{array}\right]
\end{gathered}
$$

with $x_{0}=[78.2652 .42-8.71-96.30]^{\mathrm{T}}$ and the system is perturbed to the state $x_{30}=\left[\begin{array}{llll}-12.32 & 3.4 & 8.1 & -7.965\end{array}\right]^{\mathrm{T}}$. The simulation was run with $\eta_{k}=\hat{\eta}_{k}$. Figure 2 shows the open-loop and closed-loop performance using the adaptive disturbance rejection algorithm of Section 2. Figure 3 shows $\hat{\eta}_{k}$ for the same simulation.

\section{EXPERIMENTAL RESULTS}

In this section we implement the discrete-time adaptive algorithm with integral control for angular positioning of a Maxon brushless DC motor. The objective is to rotate a steel disc mounted on the shaft through a prescribed angle. The motor is driven in torque mode by a Copley Controls amplifier, which receives a voltage command from the controller. The disc inertia, motor inertia, motor torque-current ratio and current amplifier gain are all unknown. The controller is implemented on a dSPACE DS1103 system as a C-coded Simulink S-function.

Let the angular position of the disc be denoted by $\theta(t)$ and define the state vector $x(t) \triangleq\left[\begin{array}{c}\theta(t) \\ \dot{\theta}(t)\end{array}\right]$. The system dynamics are given by the continuous-time state space model

$$
x(t)=\left[\begin{array}{ll}
0 & 1 \\
0 & 0
\end{array}\right] x(t)+\left[\begin{array}{c}
0 \\
\frac{K_{\text {amp }} K_{\text {motor }}}{J}
\end{array}\right](V(t)+\phi)
$$

where $V(t)$ is the voltage input to the current amplifier, $\phi$ is an unknown constant input bias, $K_{\text {amp }}$ is the amplifier gain, $K_{\text {motor }}$ is the torque constant of the motor, and $J$ is the total inertia of the disc and the motor armature.

Defining $\mathscr{K} \triangleq K_{\text {amp }} K_{\text {motor }} / J$ and discretizing (64) using a zero-order hold equivalent at a sampling rate of $T$, we obtain

$$
x_{k+1}=A x_{k}+B V_{k}+B \phi
$$




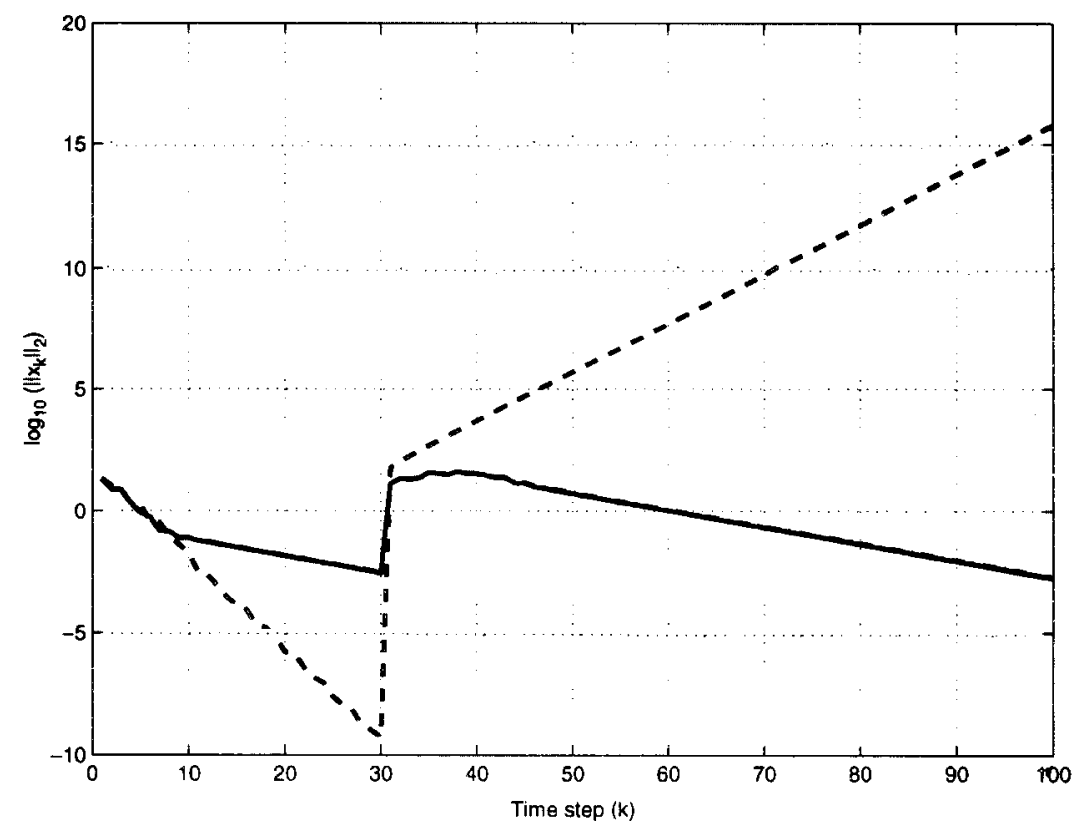

Figure 2. Open-loop and closed-loop performance from numerical simulation. Dashed line: open-loop, solid line: closed-loop.

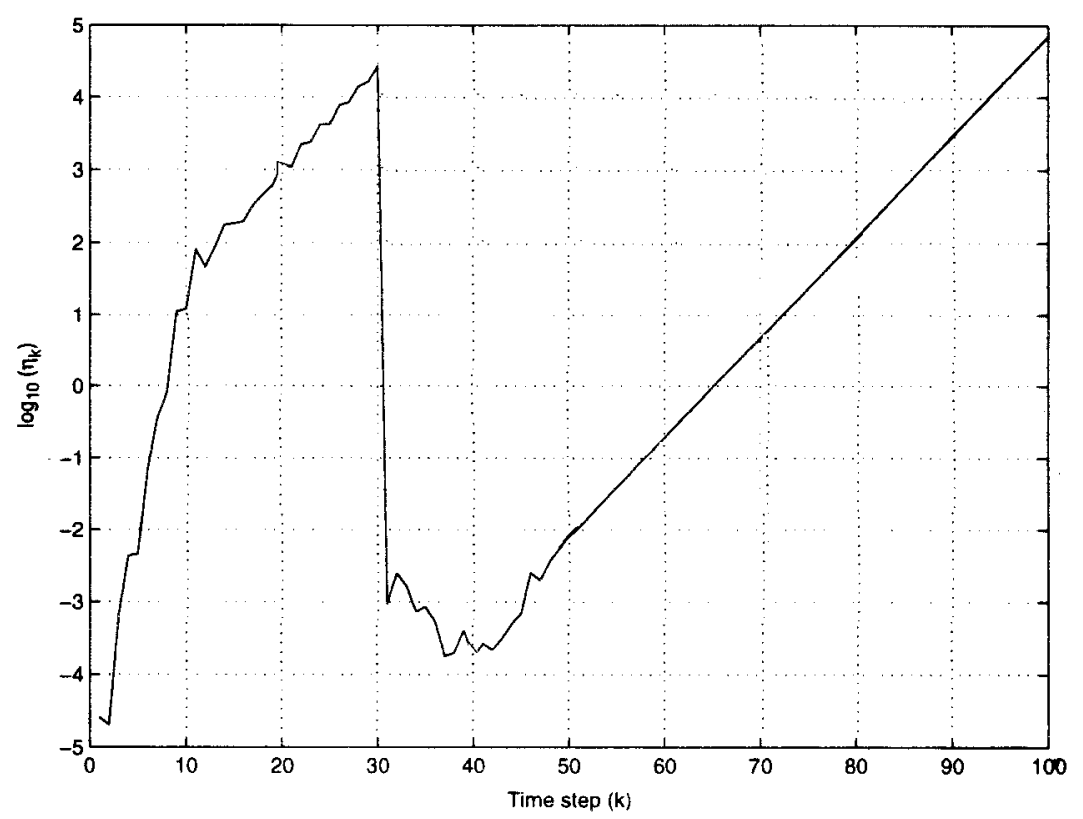

Figure 3. $\hat{\eta}_{k}$ from numerical simulation. 
where

$$
A \triangleq\left[\begin{array}{ll}
1 & T \\
0 & 1
\end{array}\right], \quad B \triangleq\left[\begin{array}{c}
\mathscr{K} T^{2} / 2 \\
\mathscr{K} T
\end{array}\right]
$$

We note that $\mathscr{K}$ is unknown, and that the disc angular position and angular velocity are fed back to the controller to allow full-state-feedback control. The control objective is to asymptotically drive the system to a reference state $x_{\text {ref }} \triangleq\left[\begin{array}{c}\theta_{\text {ref }} \\ 0\end{array}\right]$, that is, to rotate the disc to an angular position $\theta_{\text {ref }}$.

Next, we note that the reference state satisfies the constant state tracking condition

$$
\left(A-I_{n_{x}}\right) x_{\text {ref }}+B u_{\text {ref }}=0
$$

with $u_{\text {ref }}=0$. Subtracting (67) from (65) we obtain

$$
x_{k+1}-x_{\text {ref }}=A\left(x_{k}-x_{\text {ref }}\right)+B V_{k}+B \phi
$$

or

$$
e_{k+1}=A e_{k}+B V_{k}+B \phi
$$

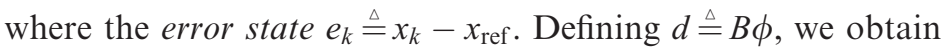

$$
e_{k+1}=A e_{k}+B u_{k}+d
$$

We observe that (70) has the same form as (36), and hence we can use the adaptive integral control algorithm of Theorem 2, which implies $\lim _{k \rightarrow \infty} e_{k}=0$.

The control algorithm is implemented as follows. First, we specify $A_{\mathrm{s}}$ to calculate $P$. Let $K_{\mathrm{s}}=\left[\begin{array}{ll}K_{1 \mathrm{~s}} & K_{2 \mathrm{~s}}\end{array}\right]$. Then, from (66) it follows that

$$
A_{\mathrm{s}}=A+B K_{\mathrm{s}}=\left[\begin{array}{cc}
1+T \alpha_{1} & T+\frac{T \alpha_{2}}{2} \\
\alpha_{1} & 1+\alpha_{2}
\end{array}\right]
$$

where $\alpha_{1} \triangleq T \mathscr{K} K_{1 \mathrm{~s}}$ and $\alpha_{2} \triangleq T \mathscr{K} K_{2 \mathrm{~s}}$. The coefficients of the second-order characteristic polynomial of $A_{\mathrm{s}}$ depend on $\alpha_{1}$ and $\alpha_{2}$, and thus, we can specify $\alpha_{1}$ and $\alpha_{2}$ to ensure that $A_{\mathrm{s}}$ is asymptotically stable even though $\mathscr{K}$ is unknown. The constants $\alpha_{1}$ and $\alpha_{2}$ are chosen such that the eigenvalues of $A_{\mathrm{s}}$ correspond to a damping ratio of 0.85 and a natural frequency of $10 \mathrm{rad} / \mathrm{s}$ with $T=0.01 \mathrm{~s}$. $A_{\mathrm{s}}$ is used to determine $P$ and to calculate $\varepsilon_{k}$. $\lambda$ is chosen to be 0.001 .

Next, from (66) we note that

$$
B=b_{0}\left[\begin{array}{c}
\frac{T}{2} \\
1
\end{array}\right]
$$

where $b_{0} \triangleq T \mathscr{K} . \mathscr{K}$ is assumed to be positive, and, although $b_{0}$ is unknown, the adaptive algorithm is robust to uncertainty in $b_{0}$ with Figures. 4-7 showing results of two tests with $b_{0}$ chosen to be 1 and 50 .

\section{CONCLUSIONS}

In this paper we derived a discrete-time adaptive stabilization algorithm and proved closed-loop attractivity with respect to the plant states. Single and multiple input cases were simulated 


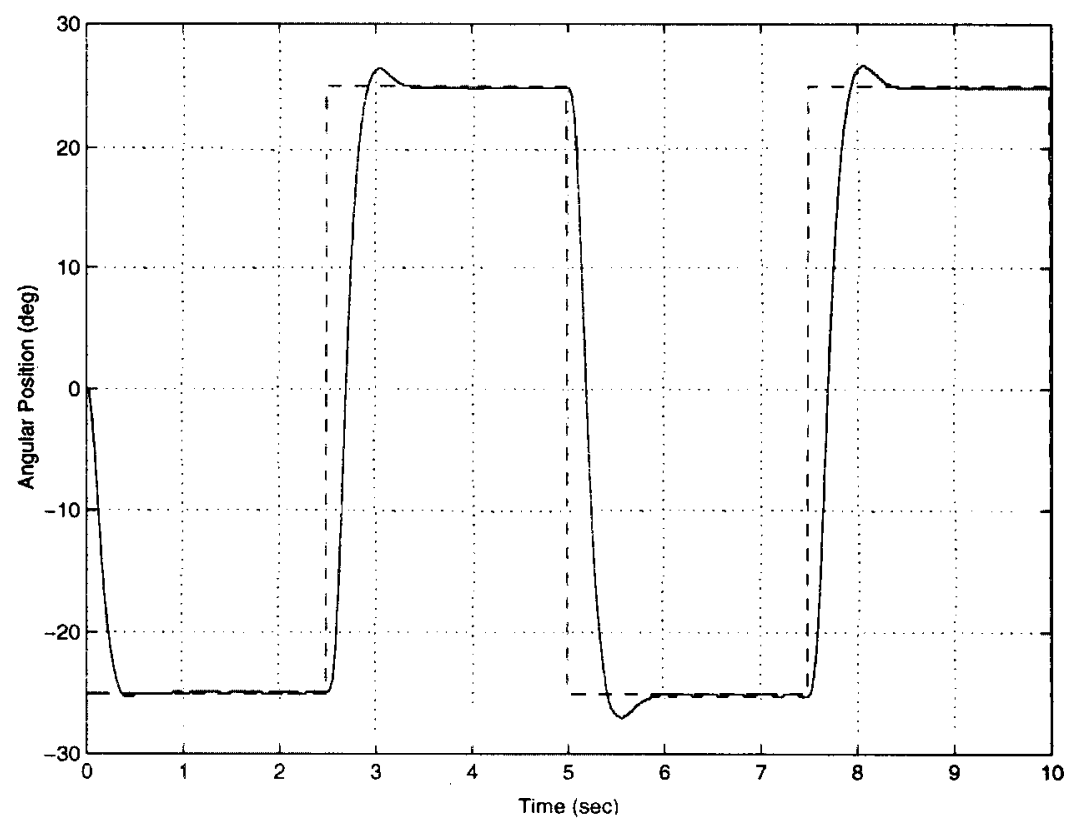

Figure 4. Angular position of disk for $b_{0}=1$. Solid line: $\theta_{k}$, dashed line: $\theta_{\text {ref }}$.

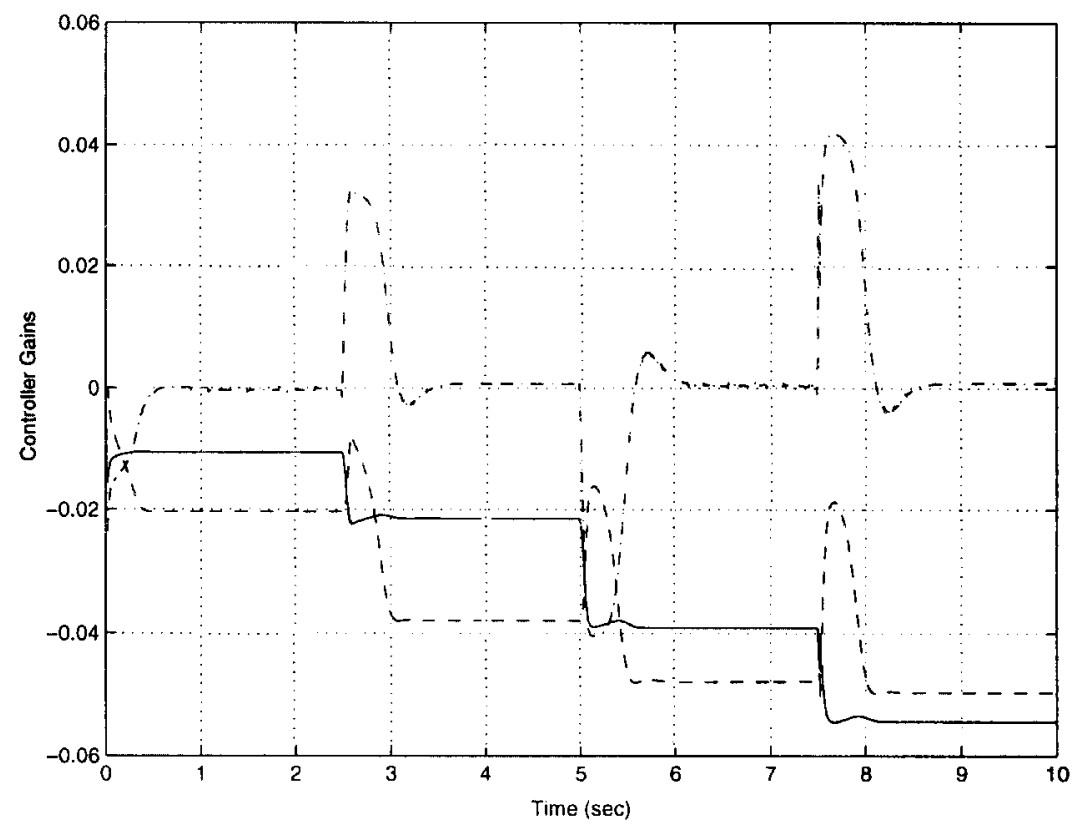

Figure 5. Controller gains for $b_{0}=1$. Solid line: $K_{1 k}$, dashed line: $K_{2 k}$, dash-dot line: $v_{k}$. 


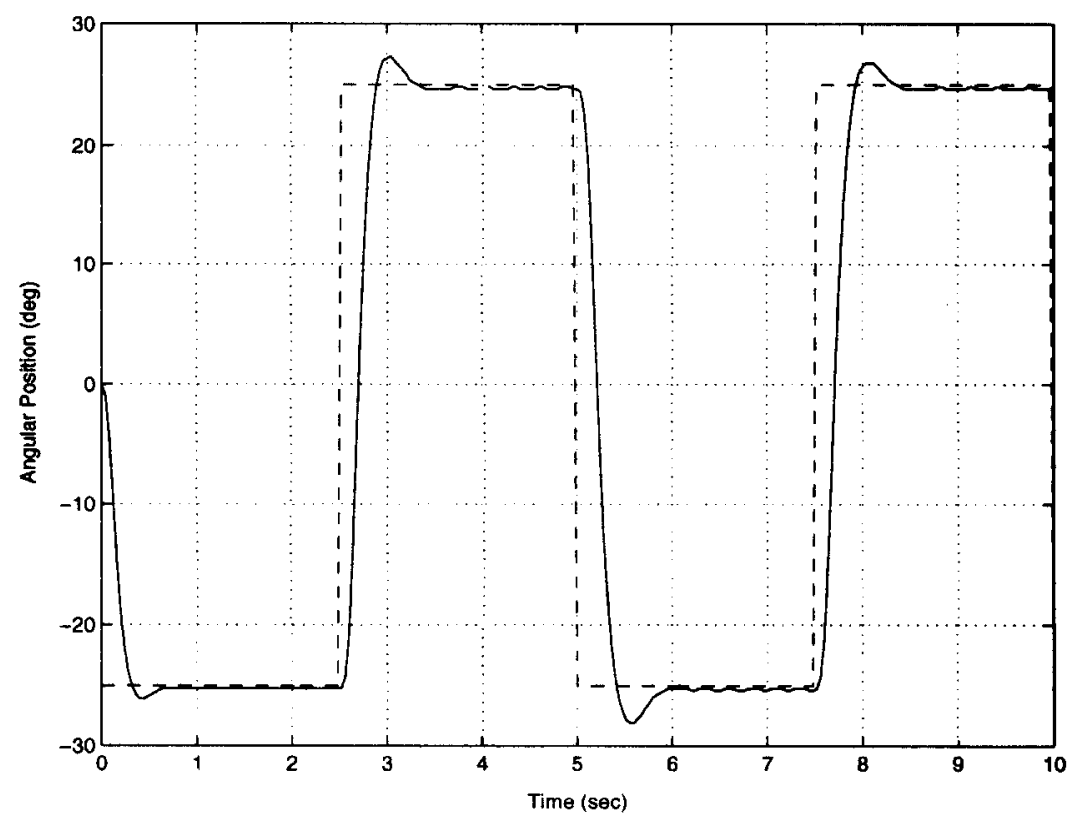

Figure 6. Angular position of disk for $b_{0}=50$. Solid line: $\theta_{k}$, dashed line: $\theta_{\text {ref }}$.

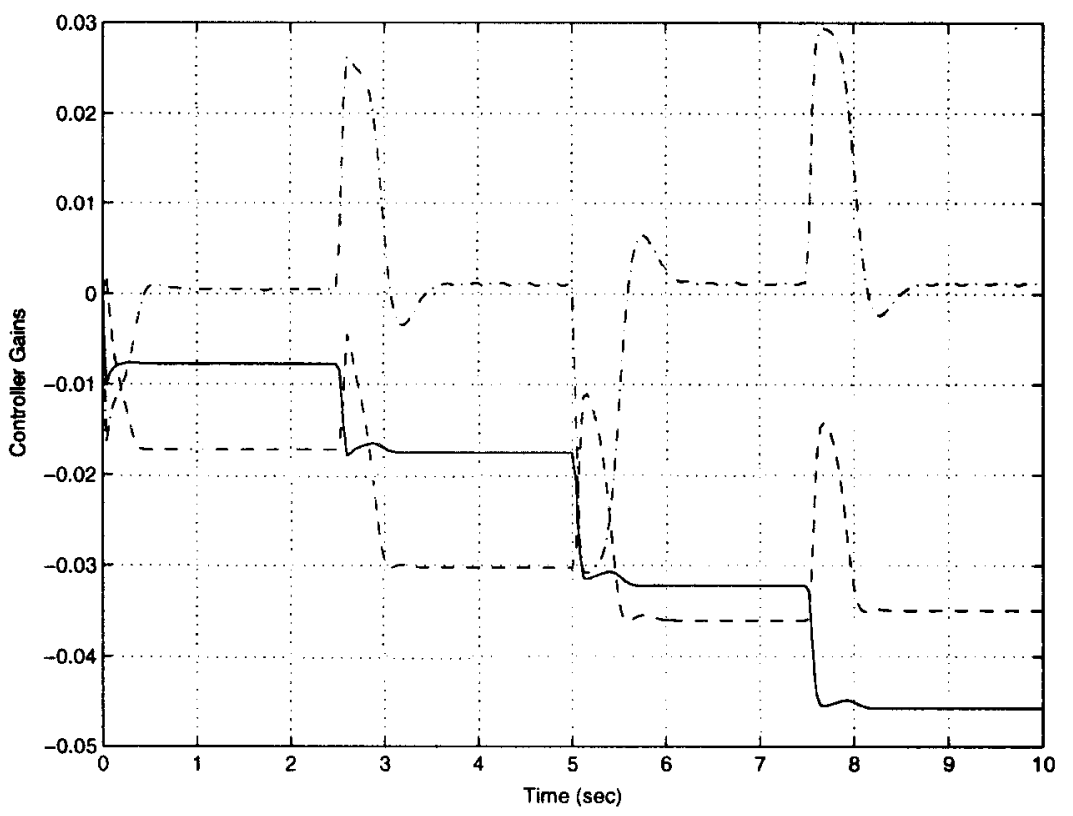

Figure 7. Controller gains for $b_{0}=50$. Solid line: $K_{1 k}$, dashed line: $K_{2 k}$, dash-dot line: $v_{k}$. 
numerically for unstable and abruptly varying plants, and experimental results were obtained on a motor positioning system. Future work will involve extensions to output feedback.

\section{ACKNOWLEDGEMENTS}

This research was supported in part by the Air Force Office of Scientific Research under grand F49620-01$1-0094$.

\section{REFERENCES}

1. Astrom KJ, Wittenmark B. Adaptive Control. Addison-Wesley: Reading, MA, 1995.

2. Ioannou PA, Sun J. Robust Adaptive Control. Prentice-Hall: Englewood Cliffs, NJ, 1996.

3. Kaufmann H, Barkana I, Sobel K. Direct Adaptive Control Algorithms: Theory and Applications. Springer: Berlin, 1998.

4. Mareels I, Polderman JW. Adaptive Systems: An Introduction. Birkhauser: New York, 1996.

5. Narendra KS, Annaswamy AM. Stable Adaptive Systems. Prentice-Hall: Englewood Cliffs, NJ, 1989.

6. Goodwin GC, Ramadge PJ, Caines PE. Discrete-time multivariable adaptive control. IEEE Transactions on Automatic Control 1980; 25:449-456.

7. Goodwin GC, Long RS. Generalization of results on multivariable adaptive control. IEEE Transactions on Automatic Control 1980; 25:1241-1245.

8. Narendra KS, Lin Y-H. Stable discrete adaptive control. IEEE Transactions on Automatic Control 1980; 25:456-461.

9. Fuchs J-J. Discrete adaptive control: a sufficient condition for stability and applications. IEEE Transactions on Automatic Control 1980; 25:940-945.

10. Egardt B. Unification of some discrete-time adaptive control schemes. IEEE Transactions on Automatic Control 1980; 25:693-697.

11. Egardt B. Stability analysis of discrete-time adaptive control schemes. IEEE Transactions on Automatic Control 1980; 25:710-715.

12. Goodwin GC, Sin KS. Adaptive Filtering, Prediction and Control. Prentice-Hall, Englewood, Cliffs, NJ, 1984.

13. Landau ID, Lozano R, M'Saad M. Adaptive Control. Springer: Berlin, 1998.

14. Goodwin GC, Sin KS. Adaptive control of nonminimum phase systems. IEEE Transactions on Automatic Control 1981; 26:478-483.

15. Goodwin GC, Sin KS. Global convergence for adaptive one-step-ahead optimal controllers based on input matching. IEEE Transactions on Automatic Control 1981; 26:1269-1273.

16. Goodwin GC, Chan SW. Model reference adaptive control of systems having purely deterministic disturbances. IEEE Transactions on Automatic Control 1980; 28:855-858.

17. Mudgett DR, Morse AS. Adaptive stabilization of a discrete linear system with an unknown high-frequency gain. IEEE Transactions on Automatic Control 1980; 30:798-799.

18. Lee T-H, Narendra KS. Stable discrete adaptive control with unknown high-frequency gain. IEEE Transactions on Automatic Control 1980; 31:477-479.

19. Kanellakopoulos I. A discrete-time adaptive nonlinear system. IEEE Transactions on Automatic Control 1994; 39:1262-1265.

20. Johansson R. Global lyapunov stability and exponential convergence of direct adaptive control. International Journal of Control 1989; 50:859-869.

21. Johansson R. Supermartingale analysis of minimum variance adaptive control. Control Theory and Advanced Technology 1995; 10:993-1013.

22. Venugopal R, Bernstein DS. Adaptive disturbance rejection using AR-MARKOV/Toeplitz models. IEEE Transactions on Control System Technology 2000; 8:257-269.

23. Johnson CR, Tse E. Adaptive implementation of one-step-ahead optimal control via input matching. IEEE Transactions on Automatic Control 1978; 23:865-872.

24. Spooner JT, Ordonez R, Passino K. 'Direct adaptive fuzzy control for a class of discrete-time systems. Proceedings of the American Control Conference June 1997; 1814-1818.

25. Nounou HN, Passino KM. Stable auto-tuning of the adaptation gain for direct adaptive control. Proceedings of the American Control Conference June 2000; 2154-2158.

26. Nounou HN, Passino KM. Stable auto-tuning of the adaptation gain for indirect adaptive control. Proceedings of the American Control Conference June 2000; 2159-2163.

27. La Salle JP. The Stability of Dynamical Systems. SIAM: Philadelphia, PA, 1976. 